\title{
A Comparison of Nanometrology Using TEM and SEM
}

\author{
J. B. Warren * and B. J. Panessa-Warren** \\ * Center for Functional Nanomaterials and Instrumentation Divison, Brookhaven National \\ Laboratory, Upton, NY, 11973 \\ ** Dept of Materials Science, Brookhaven National Laboratory, Upton, NY, 11973
}

Great efforts have been expended to develop reliable methods to measure nanoscale linewidths using scanning electron microscopy. SEM-based instruments dedicated to linewidth measurement are commercially available and their limitations have been well characterized [1]. However, continuing efforts to design semiconductor devices with a critical dimension of $65 \mathrm{~nm}$ and smaller focuses attention on alternative metrological methods that can potentially provide superior resolution.

This paper compares the nanoscale measurement capabilities of a modern JEOL 6500 field emission SEM, and an older generation Philips 300 TEM with a high-resolution stage configuration. For optimum conditions, the Philips 300 has a point-to-point resolution of $0.25 \mathrm{~nm}$ at $100 \mathrm{keV}$ while the JEOL 6500's resolution is $1.5 \mathrm{~nm}$ at $15 \mathrm{keV}$. It is apparent that TEM methods offer an improvement in resolution over the SEM at least by a factor of 6. Although TEM sample preparation methods will always be more time consuming than comparable methods for the SEM, the increasing use of focused ion beam (FIB) methods to rapidly thin samples to electron beam transparency will reduce TEM sample preparation times.

For a resolution comparison of the two instruments, a Nabity NPGS electron beam pattern generator interfaced with the JEOL 6500 was used to expose a concentric circular array pattern with $50 \mathrm{~nm}$ linewidths in a polymethylmethacrylate (PMMA) film $90 \mathrm{~nm}$ thick spun over a $\mathrm{Si}_{3} \mathrm{~N}_{4} \mathrm{membrane}$ The $\mathrm{Si}_{3} \mathrm{~N}_{4}$ membrane is attached to a silicon frame small enough to be mounted in a typical TEM sample holder. After exposure at $30 \mathrm{keV}$ with a dose of $900 \mathrm{uC} / \mathrm{cm}^{2}$, the PMMA pattern was developed in a 3:1 IPA:MIBK solution, and coated with a $12 \mathrm{~nm}$ layer of Cr using an Edwards 306A vacuum evaporator. The PMMA was then dissolved in acetone using the lift-off technique to fabricate the $\mathrm{Cr}$ test pattern. Finally, the $\mathrm{Cr}$ ring array was imaged with both the JEOL 6500 and the Philips 300. Traditional methods of sample preparation for high resolution SEM imaging use a $\mathrm{Cr}$ or Pt coating $3 \mathrm{~nm}$ in thickness to minimize charging and increase secondary electron yield. Since this would increase the line widths of the pattern by approximately $11 \%$ as well as reducing the contrast between the $\mathrm{Cr}$ lines and the $\mathrm{Si}_{3} \mathrm{~N}_{4}$ membrane, the sample was not coated.

As expected, the TEM image (Fig. 2) demonstrates superior resolution. Examination of the faint contrast modulation pattern in the $\mathrm{Si}_{3} \mathrm{~N}_{4}$ membrane in both the SEM and TEM images shows that the same sample location is imaged for both modes. Individual $\mathrm{Cr}$ grains in the rings are resolved in the TEM image and the boundary between the $\mathrm{Cr}$ rings and the $\mathrm{Si}_{3} \mathrm{~N}_{4}$ membrane is sharp and distinct. Efforts are now underway to determine linewidths from the digitized TEM negatives scanned at 8000 pixels/inch using statistical methods based on the AnalySIS software package. 


\section{References}

[1] A. Sicignano et al., Designing a Reference for CD-SEM Magnification Calibration, Metrology, Inspection, and Process Control for Microlithography, Proc. of SPIE Vol. 5038 (2003), pp.1123-1130.

[2] This research was supported by Dept. of Energy Contract No. DE-AC0298CH10886.

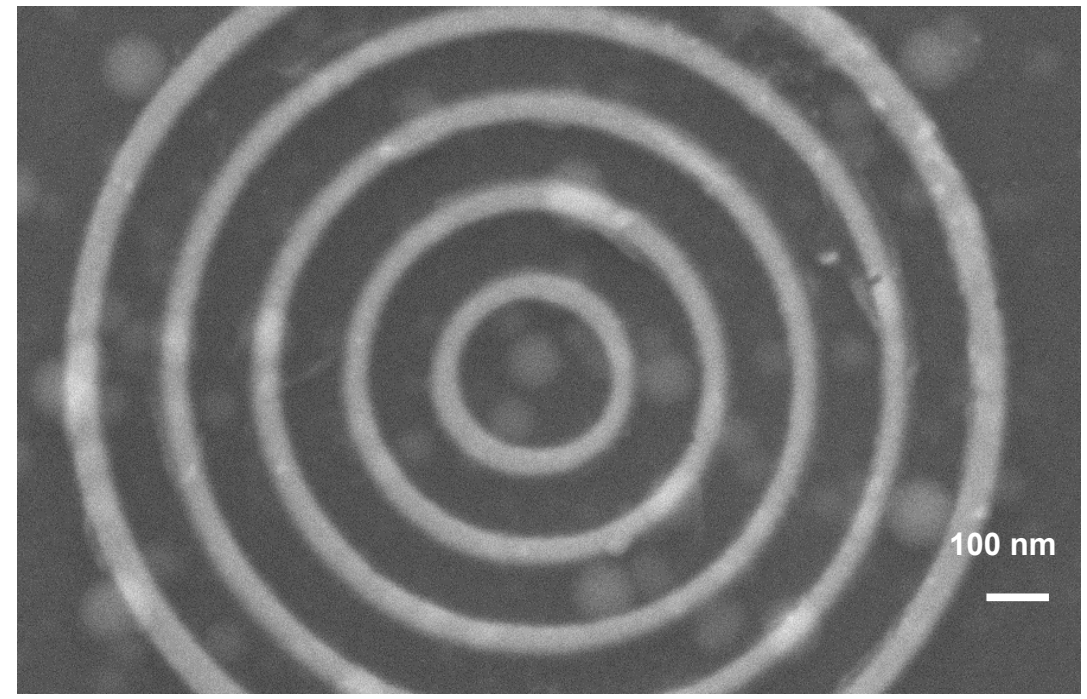

FIG. 1. JEOL 6500 secondary electron image at $15 \mathrm{keV}$ of $\mathrm{Cr}$ ring pattern on a $\mathrm{Si}_{3} \mathrm{~N}_{4}$ membrane fabricated by e-beam lithography. Outer ring linewidth is $50 \mathrm{~nm}$, inner rings are $30 \mathrm{~nm}$. Sample is uncoated.

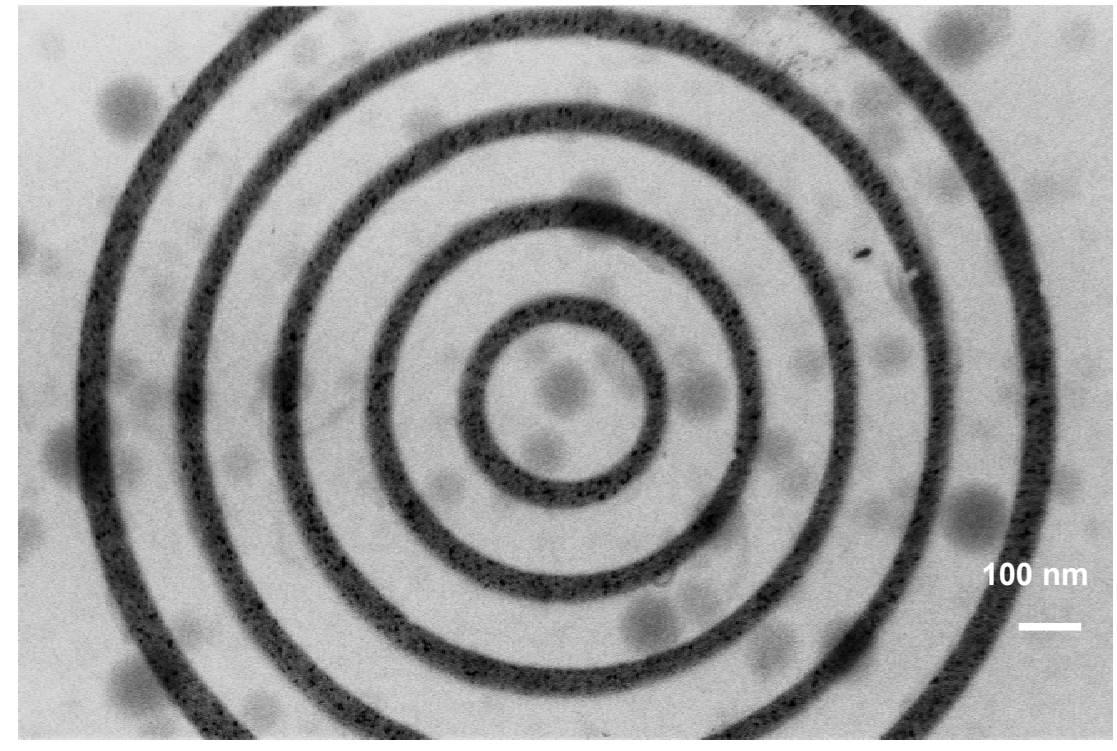

FIG. 2. Philips $300 \mathrm{TEM}$ positive image of the same $\mathrm{Cr}$ ring pattern at $100 \mathrm{keV}$. The original negative has been scanned at reduced resolution of 500 pixels per inch to minimize file size for publication. 